\title{
DYNAMICAL HISTORY OF THE OORT CLOUD
}

\author{
Paul R. Weissman \\ Earth and Space Sciences Division \\ Jet Propulsion Laboratory \\ 4800 Oak Grove Drive \\ Pasadena, CA 91109 USA
}

\begin{abstract}
Dynamical studies during the past decade have resulted in an almost explosive increase in our understanding of the Oort cloud of comets, which surrounds the solar system. Cometary orbits in the cloud evolve under the complex interaction of stellar, galactic, and giant molecular cloud perturbations, as well as planetary and nongravitational perturbations when the orbits re-enter the planetary region. Evidence has continued to build for a dense, inner Oort cloud of comets which acts as a reservoir to replenish the outer cloud as comets there are stripped away. A ring of comets beyond the orbit of Neptune, which may be the source of the short-period comets, is also likely. Both the estimated number and mass of comets in the Oort cloud have grown such that the total mass may be comparable to the mass of the planets. Temporal variations in the flux of comets from the Oort cloud into the planetary region by a factor of $50 \%$ are typical, and by factors of 20 to 200 are possible. The most intense cometary "showers" may have serious implications for biological extinction events on Earth as well as for the impact history of planets and satellite systems. Comets in the Oort cloud are processed by galactic cosmic rays, heated by nearby supernovae, eroded by interstellar dust impacts, and disrupted by mutual collisions (in the inner cloud). A detailed estimate of the Oort cloud's dynamical history is not possible because of the inability to reconstruct the Sun's varying galactic motion over the history of the solar system, and because of uncertainty over where comets actually formed. However, it is likely that a substantial fraction of the original Oort cloud population has been lost to interstellar space. We are approaching the time when Oort clouds around other stars may be detectable, though searches to date have so far been negative.
\end{abstract}

\section{Introduction}

The huge spherical cloud of comets surrounding the planetary system was first suggested by Dutch astronomer Jan Oort in 1950. Oort was attempting to explain the unusual distribution of orbital energies for the observed long-period (LP) comets, shown in Figure 1 (plotted as a function of the original inverse semimajor axis, $1 / a_{o}$, which is equivalent to orbital energy). The orbital data in Figure 1 are from Marsden et al. (1978). The original orbit is the orbit of the comet integrated backwards in time to before it entered 


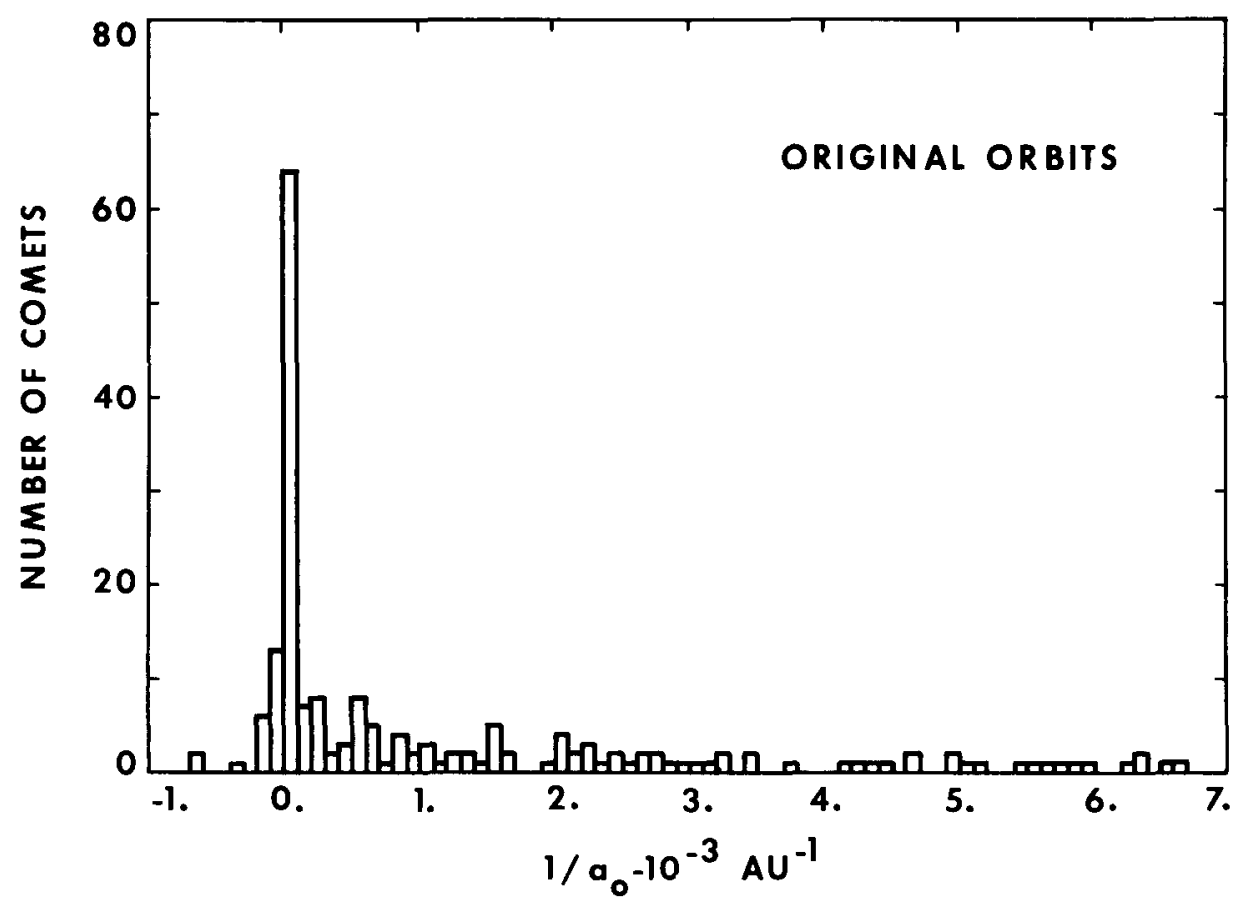

Figure 1. Distribution of original inverse semimajor axes, $1 / a_{o}$, for the observed longperiod comets. The sharp spike of comets at near-zero but bound energies are the dynamically "new" comets from the Oort cloud. The few hyperbolic comets are likely the result of errors in their orbit determinations. Orbital data from Marsden et al. (1978).

the planetary system, and referenced to the barycenter of the solar system. The $1 / a_{0}$ distribution is marked by a sharp spike of comets at near-zero but bound energies, representing orbits with semimajor axes between $10^{4} \mathrm{AU}$ and infinity; a low, continuous distribution of more tightly bound, less eccentric orbits; and a few apparently hyperbolic orbits. Earlier dynamical calculations by van Woerkom (1948) had shown that Jupiter would perturb the orbits of comets passing through the planetary system so as to randomly spread them in energy, giving rise to the low, continuous distribution. But what then was the explanation for the spike of comets?

Oort recognized that the spike had to be the source of the LP comets, a vast, spherical cloud of comets at distances greater than $10^{4} \mathrm{AU}$ from the Sun, but still gravitationally bound to it. Oort showed that comets in the cloud are so far from the Sun that distant perturbations from random passing stars can change their orbital elements and occasionally send the comets back into the planetary system. On their first pass through the planetary system, Jupiter's random perturbation ejects roughly half the "new" comets to interstellar space, while capturing the other half to more tightly bound, less eccentric orbits. Only about $5 \%$ of the new comets are returned to Oort cloud distances (Weissman, 1979). On subsequent returns the comets continue to random walk in orbital energy until they either are ejected, captured to a short-period orbit, collide with a planet or the Sun, or are 
physically destroyed by one of several poorly understood physical mechanisms. The few "hyperbolic" comets in Figure 1 are most likely the result of small errors in their orbit determinations, or unmodeled nongravitational forces resulting from jetting of volatiles on the surfaces of the cometary nuclei (which make the orbits appear more eccentric than they actually are, as shown by Marsden et al., 1973).

Oort's accomplishment in defining the source of the LP comets is even more impressive when one considers that it was based on only 19 well-determined cometary orbits, compared with the $\sim 190$ high-quality orbits in Figure 1 . Further analysis of the observed orbits by Marsden et al. (1978) shows that the average dynamically "new" LP comet entering the planetary system from the Oort cloud comes from an aphelion distance of 4.3 $\mathrm{x} 10^{4} \mathrm{AU}$.

Some researchers have pointed out that Öpik (1932) anticipated Oort's work by studying the effects of stellar perturbations on distant meteor and comet orbits 18 years earlier. Although Öpik did suggest that stellar perturbations would raise the perihelia of comets, resulting in a cloud of objects surrounding the solar system, he specifically rejected that comets in the cloud could ever be observed, because he did not recognize that stellar perturbations would also cause some orbits to diffuse back into the planetary region. Öpik concluded that the observed long-period comets came from aphelion distances of only 1,500 to 2,000 AU. In addition, he overestimated the sphere of influence of the Sun by a factor of five. Though Öpik's 1932 paper was a pioneering work on stellar perturbations, it did not identify the cometary cloud as the source of the long-period comets or relate the observed orbits to the dynamical theory.

Interestingly, speculations by Halley (1705) in his classic work on comets could be interpreted as inferring a distant comet cloud. Halley was only able to fit parabolic elements to the 24 sets of comet observations he studied, but he argued that the orbits would prove to be elliptic, writing, "For so their Number will be determinate and, perhaps, not so very great. Besides, the Space between the Sun and the fix'd Stars is so immense that there is Room enough for a Comet to revolve, tho' the Period of its Revolution be vastly long."

It is possible to simulate the dynamical evolution of comets in the Oort cloud using a computer-based Monte Carlo simulation model. The results of one such simulation by Weissman (1979) for $10^{5}$ hypothetical comets are shown in Figure 2, where the comets are perturbed by the major planets, random passing stars, and nongravitational forces, and removed by collisions, random disruption (splitting), and loss of all volatiles (sublimation of all ices). A fairly good match to the observed distribution in Figure 1 is obtained. By "tuning" such a model to improve the fit, some insight into the possible physical and dynamical loss mechanisms can also be obtained.

As a result of such modeling, it is found that $65 \%$ of the LP comets are dynamically ejected from the solar system on hyperbolic orbits, $27 \%$ are randomly disrupted $-10 \%$ on the first perihelion passage -- and the remainder are lost by a variety of processes such as loss of all volatiles and collision with the Sun and planets. The average ejection velocity is $0.6 \mathrm{~km} \mathrm{~s}^{-1}$. Some comets may become unobservable due to the formation of a nonvolatile lag deposit, or "crust," on their surfaces (Brin and Mendis, 1979), which cuts off further cometary activity. The average LP comet with perihelion $<4$ AU makes 5 passages through the planetary region before arriving at some end-state, with a mean lifetime of $6 \times 10^{5}$ years between the first and last passages.

Bailey (1984) found that the height of the low, continuous distribution of returning 


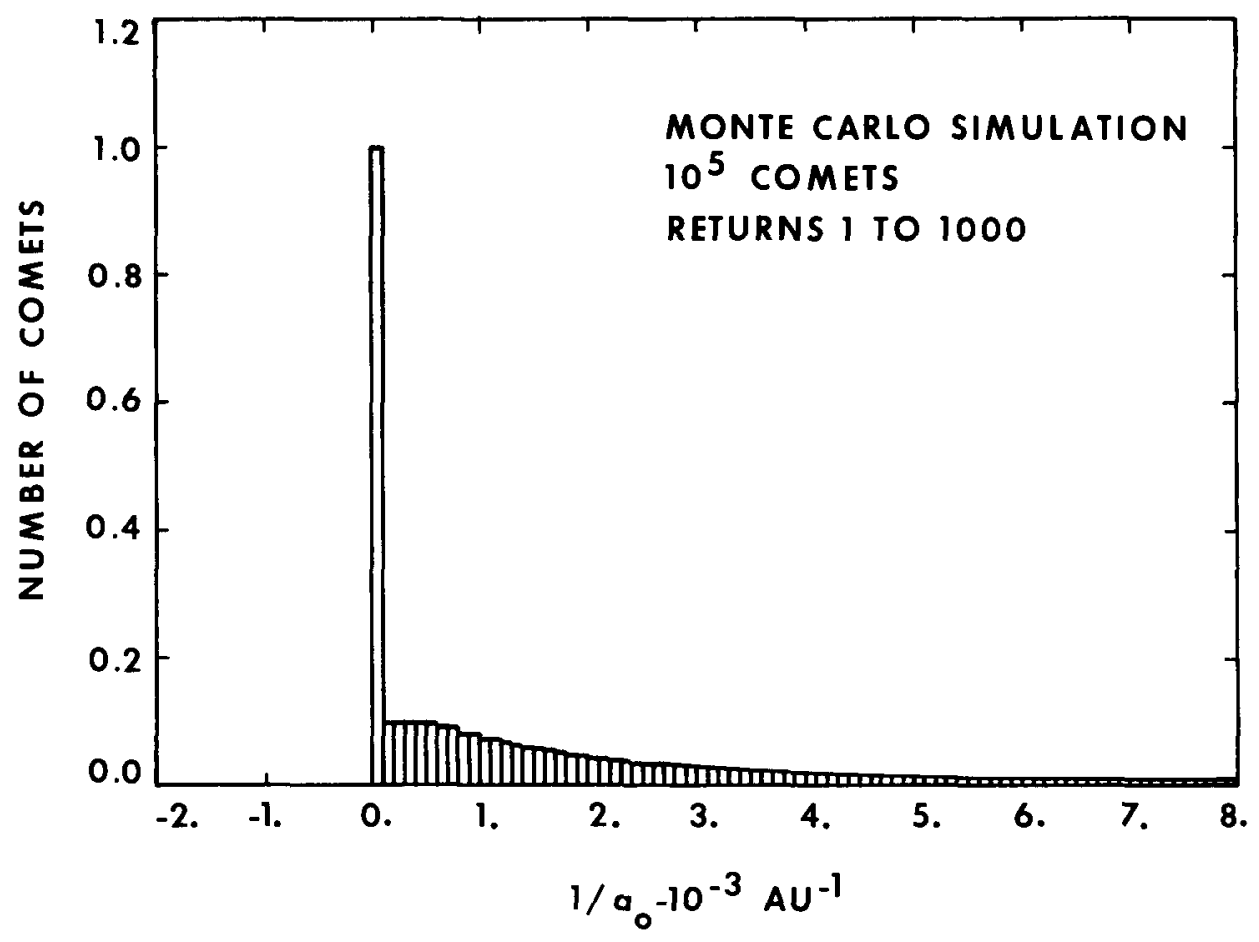

Figure 2. Estimated distribution of inverse semimajor axes for LP comets based on a Monte Carlo simulation model by Weissman (1979) that includes the effects of planetary, stellar, and nongravitational perturbations, and physical loss due to collisions, random disruption, and loss of all volatiles.

comets was too low relative to the height of the Oort cloud spike, a problem that was also commented on by Oort (1950). They each suggested that comets faded sharply after their first appearance. However, Weissman (1979) was able to achieve good agreement with the observed $1 / a_{o}$ distribution, without assuming any fading. The problem is a complex one, since Oort cloud comets on their first perihelion passage are typically anomalously bright at large heliocentric distances (Oort and Schmidt, 1951). Suggested mechanisms for this effect include a veneer of volatiles accreted from the interstellar medium and lost on the first perihelion passage near the Sun, blow-off of a primordial cosmic ray processed nucleus crust, or the amorphous-crystalline water ice phase transformation that occurs at about 5 AU inbound (Prialnik and Bar-Nun, 1987) on the first perihelion passage. When these Oort cloud comets return, they are not observed unless they come within 3 AU of the Sun, where water ice can begin to sublimate. The failure to observe returning LP comets with perihelia $>3$ AU must be an observational selection effect, since there is no recognized physical and/or dynamical mechanism for removing them.

Thus, the problem involves a combination of dynamics, physical modeling, and observational selection effects. Oort's (1950) comet sample was small and probably biased to comets with very large semimajor axes, as those were the ones that appeared hyperbolic prior to correction for planetary perturbations. Bailey (1984) uses a "corrected" 1/a 
distribution that probably over-corrected for errors in orbit determinations and shoved too many comets into the Oort cloud spike. On the other hand, Weissman (1979) may have overestimated the destructive effect of cometary splitting, since at least a few examples of disrupted comets pairs and/or families are now recognized. But when all observational and physical effects are taken into account, it is likely that "cometary fading" does not need to be invoked to explain the observed $1 / \mathrm{a}_{\mathrm{o}}$ distribution.

To account for the observed flux of dynamically "new" LP comets, Oort estimated that the population of the cometary cloud was $1.9 \times 10^{11}$ objects. More recent dynamical models (Weissman, 1982; Fernandez, 1982) have produced somewhat higher estimates, by about an order of magnitude. These result in part from higher estimates of the flux of LP comets (brighter than absolute magnitude, $H_{10}=11$ ) through the planetary system, after correction for observational selection effects as shown by Everhart (1967), and in part from a recognition of the role of the giant planets in blocking the diffusion of cometary orbits back into the planetary region (Weissman, 1985a). Comets perturbed inward to perihelia near the orbits of Jupiter and Saturn will likely be hyperbolically ejected before they can diffuse to smaller perihelia and be observed. Thus, the terrestrial planets region is undersupplied in LP comets.

\section{Oort Cloud Perturbers}

Since first proposed in 1950, Oort's vision of a cometary cloud gently stirred by perturbations from distant passing stars has evolved considerably. Additional perturbers have been recognized: giant molecular clouds (GMCs) in the galaxy, which were unknown before 1970 (Biermann, 1978; Clube and Napier, 1982); and the galactic gravitational field itself, in particular, the tidal field of the galactic disk (Byl, 1983; Harrington, 1985; Heisler and Tremaine, 1986). GMC encounters are rare, occurring with a mean interval of $3 \times 10^{8}$ years, but result in major perturbations on the orbits in the Oort cloud. The galactic field sets the limits on the outer dimensions of the Oort cloud. The cloud is a prolate spheroid with the long axis oriented toward the galactic nucleus (Antonov and Latyshev, 1972; Smoluchowski and Torbett, 1984). Maximum semimajor axes are about $10^{5} \mathrm{AU}$ for direct orbits (relative to galactic rotation) oriented along the radius vector, decreasing to about $8 \times 10^{4} \mathrm{AU}$ for orbits perpendicular to the galactic radius vector, and increasing to $1.2 \mathrm{x}$ $10^{5} \mathrm{AU}$ for retrograde orbits (opposite to galactic rotation).

In addition, random stars will occasionally pass directly through the Oort cloud, ejecting a substantial number of the comets and severely perturbing the orbits of others (Hills, 1981). A star passage drills a narrow tunnel through the Oort cloud, ejecting all comets within a radius of $\sim 450 \mathrm{AU}$, for a $1 \mathrm{M}_{\odot}$ passing at a velocity of $20 \mathrm{~km} \mathrm{~s}^{-1}$ (Nezhinskij, 1972; Weissman, 1980). Over the history of the solar system, $\sim 5.4 \times 10^{3}$ stars have passed within $10^{5} \mathrm{AU}$ of the Sun, ejecting about $10 \%$ of the Oort cloud population.

It is now recognized that the galactic disk is the major perturber of the Oort cloud, though stars and GMCs still play an important role in repeatedly randomizing the cometary orbits. Galactic tidal perturbations peak for orbits with their line of apsides at galactic latitudes of $\pm 45^{\circ}$ and go to zero at the galactic equator and poles. Delsemme (1987) showed that the distribution of galactic latitudes of the aphelion directions of the observed LP comets mimics that dependence, as shown in Figure 3. Although a lack of comet discoveries near the galactic equator could be the result of observational selection effects 


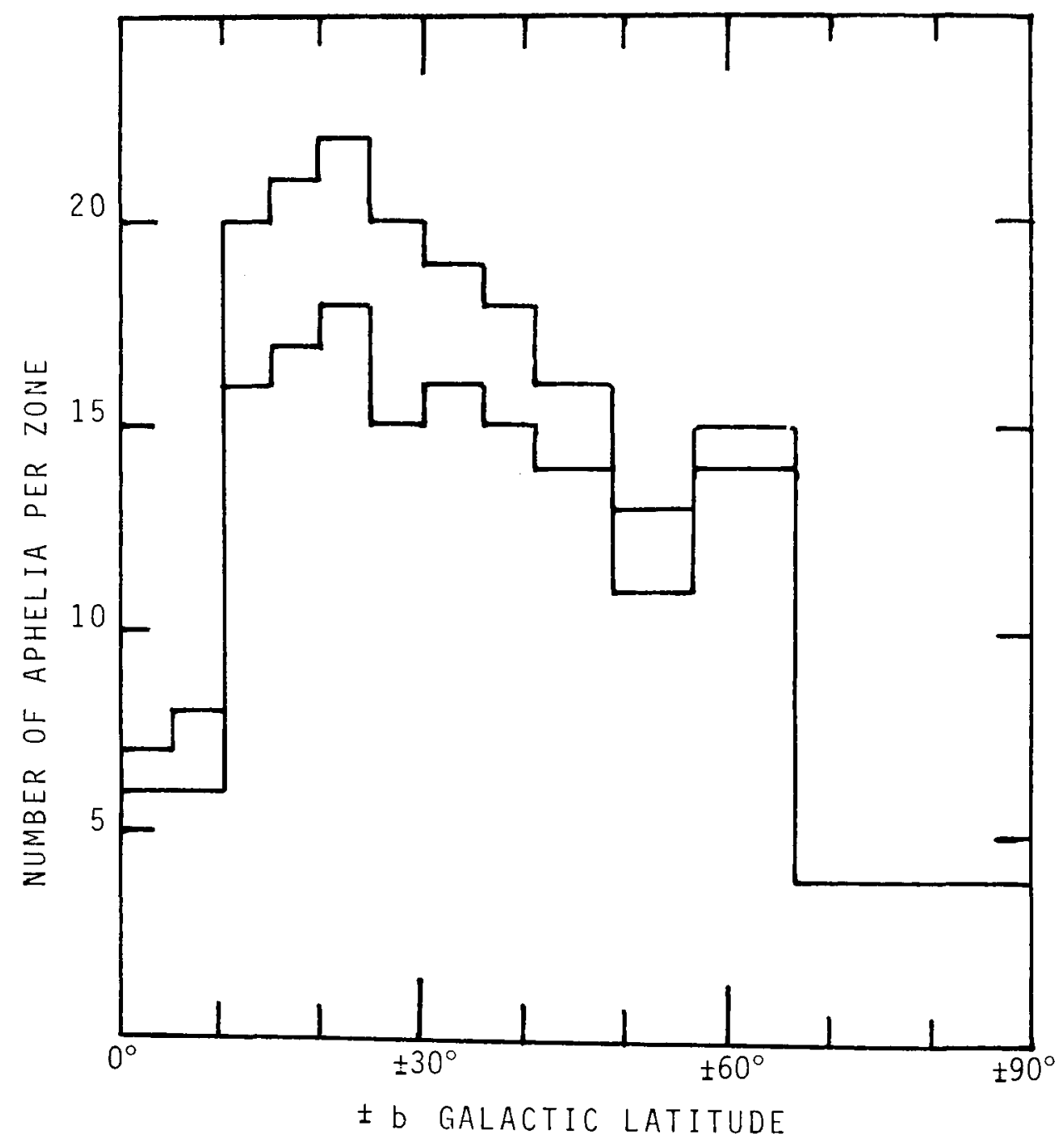

Figure 3. Distribution in galactic latitude of aphelion directions for 152 long-period comets with orbital periods $>10^{4}$ years, plotted in bins of equal area on the sky (Delsemme, 1987). Comets are deficient at the galactic equator and poles, in agreement with the expectation from galactic tides acting on the Oort cloud. The lower histogram is the raw data; the upper histogram includes a correction for the lack of Southern hemisphere observers.

(confusion with galactic nebulae), the lack of comets near the poles appears to confirm the importance of the galactic field on the Oort cloud.

Because the galactic tide acts to step the cometary perihelia in a regular fashion, rather than the random walk nature of the stellar perturbations, comets are brought into the observable region far more efficiently, making it easier to overcome the dynamical barrier 
that Jupiter and Saturn present to cometary diffusion into the inner planets region. As a result, estimates of the population of the Oort cloud should decrease, though detailed estimates with a complete model have yet to be made. One Monte Carlo simulation by Heisler et al. (1987) suggests that the decrease might be as much as a factor of four, or a corrected population of less than $\sim 5 \times 10^{11}$ comets. However, that model did not include the perturbing effect of the major planets.

\section{The Inner Oort Cloud}

As a result of this better understanding of the competing roles of Oort cloud perturbers, it is now estimated that the mean dynamical lifetime of comets in the cloud is only about $60 \%$ the age of the solar system (Hut and Tremaine, 1985), though some authors have estimated shorter lifetimes (Bailey, 1986). The Oort cloud must somehow be replenished, either by capture of comets from interstellar space, as suggested by Clube and Napier (1984), or from a more populous inner Oort cloud reservoir, comets in orbits closer to the Sun that are pumped up to replace the lost comets, as suggested by Hills (1981) and even admitted to by Clube and Napier (1984).

Cometary capture is a highly unlikely process because a three-body gravitational interaction is required to dissipate the excess hyperbolic energy. The possibility of capture has been shown by Valtonen and Innanen (1982) and Valtonen (1983) to be proportional to $V_{\infty}{ }^{-7}$, where $V_{\infty}$ is the hyperbolic excess velocity. Capture is possible at encounter velocities $\leq 1 \mathrm{~km} \mathrm{~s}^{-1}$, but is highly unlikely at the Sun's velocity of $16.5 \mathrm{~km} \mathrm{~s}^{-1}$ relative to the Local Standard of Rest.

On the other hand, new Monte Carlo simulations by Shoemaker and Wolfe (1984) and by Duncan et al. (1987) have shown that the dynamical evolution of icy planetesimals formed in the Uranus-Neptune zone would naturally lead to a massive inner Oort cloud. The icy planetesimals would be perturbed by the forming proto-planets into eccentric longperiod orbits with semimajor axes of several times $10^{3} \mathrm{AU}$. Galactic and stellar perturbations at aphelion would be sufficient to raise the perihelia of such orbits to heliocentric distances greater than the semimajor axis of the orbit of Neptune, detaching the comets from planetary perturbations. In this manner, a massive inner Oort cloud reservoir, with a population between five and ten times that of the outer cloud, could be formed. As comets are stripped away from the outer cloud by close stellar and GMC encounters, the same perturbations will pump up comets in the inner cloud to replace them.

An example of one of the Monte Carlo simulations by Duncan et al. (1987) is shown in Figure 4 for four different times in the solar system's history. Shown in the figure are the location of hypothetical comets in the Oort cloud, projected onto a plane perpendicular to the plane of the galaxy. Initially the comets are confined to the ecliptic plane (which is inclined approximately $60^{\circ}$ to the galactic plane). As perturbations drive the orbits outward, their inclinations slowly increase. At the end of $4.5 \times 10^{9}$ years, the cloud is essentially randomized in both inclination and eccentricity beyond about $10^{4} \mathrm{AU}$ from the Sun. However, inward of $10^{4} \mathrm{AU}$, the cloud remains flattened toward the ecliptic plane.

Approximately $40 \%$ of the original comets ejected to the Oort cloud (both inner and outer) are lost over the history of the solar system in the Duncan et al. simulation. Comets are lost due to direct ejection from close stellar passages, due to perturbation back into the 


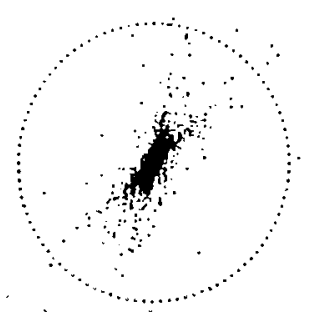

Figure 4. Dynamical evolution of a hypothetical cloud of comets ejected out of the Uranus-Neptune zone, at several times during the history of the solar system, under a combination of galactic, stellar, and planetary perturbations (projected onto a plane perpendicular to the galactic plane). The dotted circle is at a radius of $2 \times 10^{4} \mathrm{AU}$, the boundary between the inner and outer Oort clouds. From Duncan et al. (1987).

planetary region where Jupiter and Saturn perturbations will eject them hyperbolically or where they will be physically destroyed, and due to the slow growth of some orbits to distances greater than the Sun's sphere of influence, $\sim 2 \times 10^{5} \mathrm{AU}$. Most of the comets originate as planetesimals in the Uranus-Neptune zone, although a small fraction come from both the Jupiter and Saturn regions of the solar nebula. The Duncan et al. simulation did not include the effect of GMC encounters, so the fraction of comets lost should likely be looked upon as a lower limit. 
It should be recognized that the differences between the inner and outer Oort clouds are a matter of definition only. There is only one continuous cometary cloud surrounding the planetary system. The differences are defined by the different dynamical regimes: the outer Oort cloud is continuously stirred by distant stellar perturbations and the galactic tide to provide the steady-state flux of LP comets into the planetary region. In comparison, the inner Oort cloud is dynamically inactive except in the presence of major perturbers such as penetrating stellar passages and GMCs.

\section{The Population and Mass of the Oort Cloud}

Estimates of the population of the Oort cloud have been made by comparing the results of dynamical simulations of comets in the cloud with the observed flux of comets in the inner planets region. Everhart (1967) estimated that $\sim 8 \times 10^{3}$ LP comets brighter than absolute magnitude $\mathrm{H}_{10}=11$ had passed within $4 \mathrm{AU}$ of the Sun in a period of 127 years between 1840 and 1967, after correction for observational selection effects. This works out to 15.8 comets $\mathrm{AU}^{-1} \mathrm{yr}^{-1}$ if a uniform distribution of perihelia is assumed. However, Everhart (1967) also found that the LP comet perihelion distribution decreased inside of 1 AU. Bailey and Stagg (1988) and Weissman (1990) showed that only $13 \%$ to $19 \%$ of the comets with perihelia less than $4 \mathrm{AU}$ pass within $1 \mathrm{AU}$ of the Sun. Taking an average of $16 \%$, the flux of Earth-crossing $\mathrm{LP}$ comets is $10.1 \mathrm{AU}^{-1} \mathrm{yr}^{-1}$, brighter than $\mathrm{H}_{10}=11$. Absolute magnitude 11.0 corresponds to a nucleus mass of $4 \times 10^{15} \mathrm{~g}$, or a nucleus radius of $1.2 \mathrm{~km}$, assuming a density of $0.6 \mathrm{~g} \mathrm{~cm}^{-3}$ (though there is not good agreement on the cometary mass-brightness relationship; see below).

Population estimates for the Oort cloud vary from one dynamical model to another because individual researchers assume different values for the observed flux, different perturbations to be modeled, different modeling techniques, and different assumptions regarding the details of the inner and outer Oort clouds. Thus, it becomes difficult to compare such estimates. For example, Weissman (1985a) estimated an outer Oort cloud population of $2.0 \times 10^{12}$ comets brighter than $\mathrm{H}_{10}=11$ assuming a flux of 15.8 comets $\mathrm{AU}^{-1} \mathrm{yr}^{-1}$, and based on a Monte Carlo simulation that included stellar and planetary perturbations. On the other hand, Bailey and Stagg (1988) found a population for the total Oort cloud (inner plus outer) of $9.5 \times 10^{13}$ comets brighter than $\mathrm{H}_{10}=16$, based on an analytical model that included stellar and galactic tidal perturbations. Lastly, Heisler (personal communication) found a population of $0.4 \times 10^{12}$ comets brighter than $\mathrm{H}_{10}=11$, based on a flux of 10.1 comets $\mathrm{AU}^{-1} \mathrm{yr}^{-1}$ and a dynamical simulation model which included stellar and tidal perturbations.

If an attempt is made to normalize these and other results to the flux parameters determined above, then the population of the outer, dynamically active Oort cloud is in the range of $\sim 0.4 \times 10^{12}$ to $1.3 \times 10^{12}$ comets brighter than absolute magnitude $\mathrm{H}_{10}=11$. The population of the inner cloud is between five (Duncan et al., 1987) and ten (Shoemaker and Wolfe, 1984) times that of the outer cloud, or $\sim 2.0 \times 10^{12}$ to $1.3 \times 10^{13}$ comets.

The population estimates depend on the assumption that the currently observed LP comet flux through the inner planets region is equivalent to the long-term average flux. If the current flux is enhanced due to a recent perturbation on the Oort cloud, then the population estimate for the cloud is too high, and vice versa. However, Fernandez (1990) 
has pointed out that the galactic latitude dependence of the LP comet aphelion directions would not be present if most comets seen were the result of a recent major perturbation on the cloud by a close stellar passage or a GMC. Thus, the Oort cloud population estimates above may only be lower limits.

The mass of the Oort cloud is not well-known because of the uncertainties in the nucleus size distribution and in the bulk density of cometary nuclei. Estimates of the density of the nucleus of Comet Halley range from 0.2 to $1.2 \mathrm{~g} \mathrm{~cm}^{-3}$ (Rickman, 1986; Sagdeev et al., 1987; Peale, 1989), with an even larger range of uncertainties. The cometary mass distribution is even more uncertain. Most attempts at finding the mass distribution are done in two steps, first finding the distribution of cometary magnitudes, and then seeking a relationship between absolute magnitude, $\mathrm{H}_{10}$, and mass. One suggested mass-brightness relationship derived since the 1986 apparition of Comet Halley is given by Weissman (1986a, 1990)

$$
\log \mathrm{M}_{c}=20.0-0.4 \log \mathrm{H}_{10}
$$

where the nucleus mass, $M_{c}$, is in grams and a density of $0.6 \mathrm{~g} \mathrm{~cm}^{-3}$ is assumed. A similar relationship was found by Bailey and Stagg (1988), based on an average of pre- and postHalley mass-brightness relationships and an assumed density of $0.5 \mathrm{~g} \mathrm{~cm}^{-3}$

$$
\log \mathrm{M}_{\mathrm{c}}=19.9-0.5 \log \mathrm{H}_{10}
$$

Using Equation (2), the nucleus mass distribution declines more sharply as one goes to fainter magnitudes. The average nucleus mass is $3.8 \times 10^{16} \mathrm{~g}$ (for comets brighter than $\mathrm{H}_{10}$ = 11) using Equation (1), or $8.0 \times 10^{15} \mathrm{~g}$ using Equation (2). The distribution of cometary magnitudes, corrected for observational selection effects, was found by Everhart (1967) and is shown in Figure 5. The mass scale along the bottom was derived using Equation (1).

Estimates for the total cloud mass range from about $\sim 14$ to 1,000 Earth masses (Bailey and Stagg, 1988; Weissman, 1986a; Marochnik et al., 1988), with the upper limit being more than twice the total mass of the planetary system. A current best guess for the total mass, in this author's opinion, is probably $\sim 2.7 \times 10^{29} \mathrm{~g} \approx 46$ Earth masses $\left(\mathrm{M}_{\oplus}\right)$, assuming an outer Oort cloud population of $10^{12}$ comets, a total Oort cloud population of $7.0 \times 10^{12}$ comets, an average nucleus mass of $3.8 \times 10^{16} \mathrm{~g}$ (Weissman, 1990), and a nucleus bulk density of $0.6 \mathrm{~g} \mathrm{~cm}^{-3}$. Over the history of the solar system, the Oort cloud has lost between $40 \%$ (Duncan et al., 1987) and 80\% (Weissman, 1985a) of its population, so the original mass must have been about a factor of two to five larger.

The recent estimate of the Oort cloud mass by Marochnik et al. (1988) also included an estimate for the angular momentum of between $5 \times 10^{52}$ and $2 \times 10^{53} \mathrm{~g} \mathrm{~cm}^{2} \mathrm{~s}^{-1}$, far more than that of the entire planetary system. However, that estimate is likely high. It assumes a total population for the inner and outer clouds a factor of 15 greater than that given above, based only on Hills' (1981) early speculations as to what the population might be. Also, Marochnik et al.'s conclusion that this high angular momentum in the Oort cloud means that the proto-planets could not have ejected so much material without spiralling in towards the Sun is incorrect; much of the present angular momentum in the cloud comes from the action of external perturbers over the history of the solar system. The initial angular momentum must have been considerably less. 

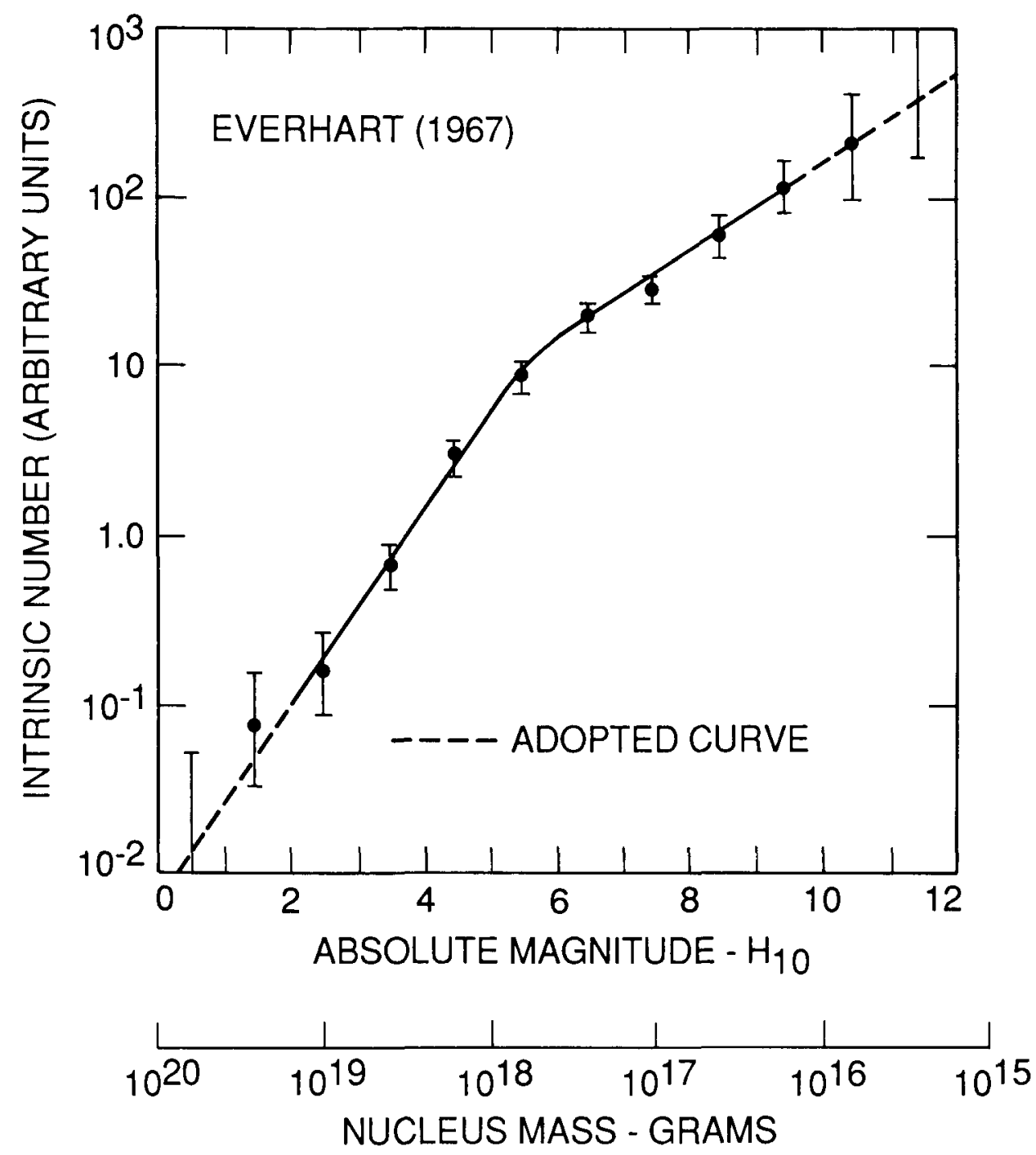

Figure 5. Relative distribution of absolute magnitudes, $\mathrm{H}_{10}$, for long-period comets after correction for observational selection effects. From Everhart (1967). The mass scale at the bottom was derived using Equation 1 (see text).

\section{Temporal Variations in the Cometary Flux}

Monte Carlo studies have shown that variations in the expected flux from the outer Oort cloud by a factor of two to three are common, and by a factor of ten may occur occasionally (Heisler et al., 1987; Fernandez and Ip, 1987). However, more recent results by Heisler (1990) have indicated that the random fluctuations in the steady-state flux from 


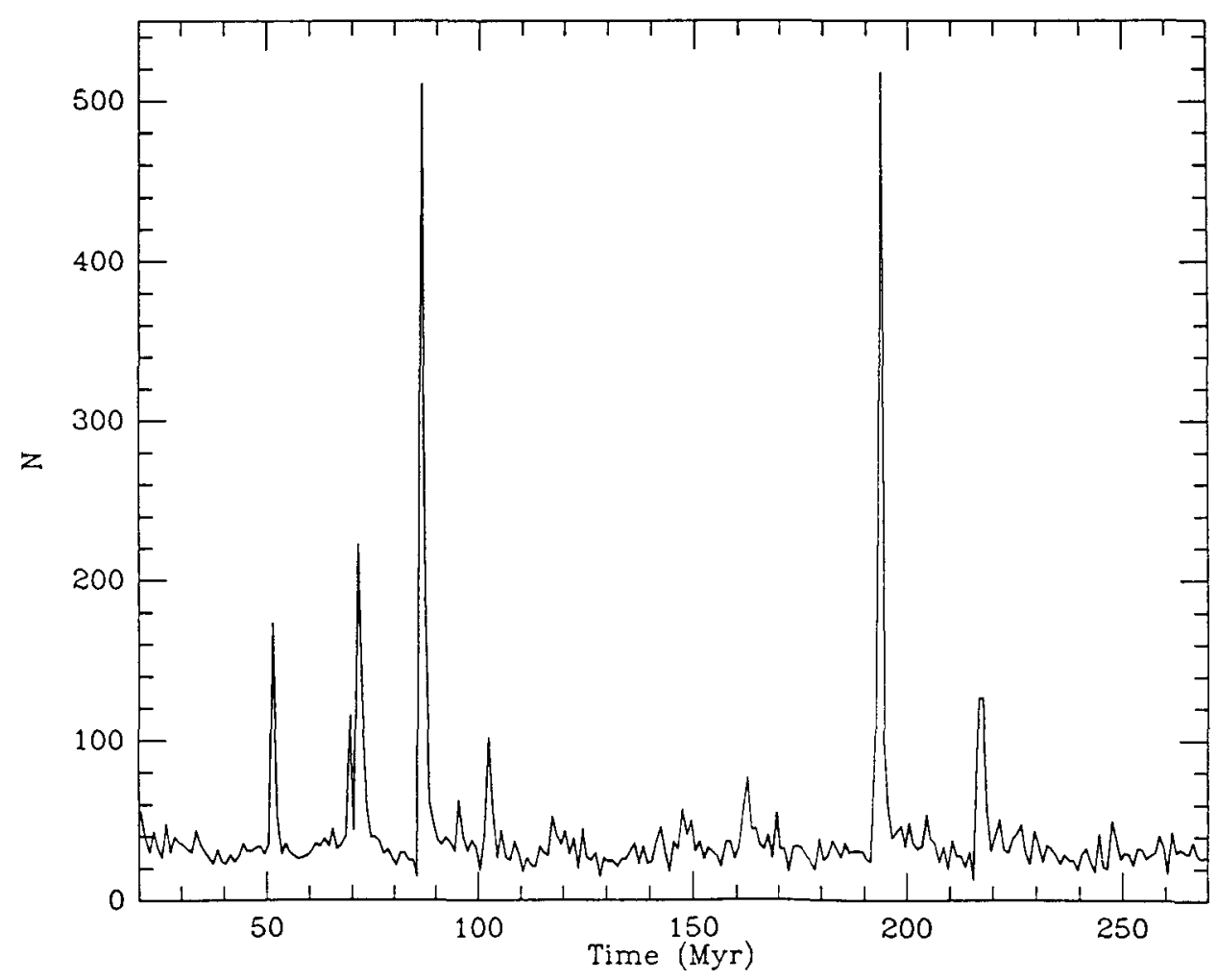

Figure 6. Number of new long-period comets from the Oort cloud entering the terrestrial planets region, $\mathrm{q}<2 \mathrm{AU}$, versus time, based on a Monte Carlo simulation that included random passing stars and galactic perturbations. The large spikes are comet showers due to random stars penetrating the Oort cloud. From Heisler (1990).

the Oort cloud are likely only $\sim 50 \%$, possibly even less. The larger variations found in the earlier Heisler et al. (1987) study tended to reflect the limited size of the statistical sample, rather than true variations in the flux. An example of the expected flux into the terrestrial planets region, $\mathrm{q}<2 \mathrm{AU}$, from one of the newer simulations by Heisler (1990) is shown in Figure 6.

More extreme variations in the flux are possible if a star passes directly through the Oort cloud, in particular, through the inner Oort cloud. Hut and Weissman (1985) used a detailed Monte Carlo simulation to show that a star passage at $3 \times 10^{3}$ AU from the Sun would perturb a shower of $\sim 5 \times 10^{8}$ comets into Earth-crossing orbits, raising the expected impact rate by a factor of 300 or more, and lasting 2 to $3 \times 10^{6}$ years. Typical comets from the inner Oort cloud each make an average of 8.5 returns in a major shower. The very high flux rate results because the shower comets from the inner Oort cloud start from shorter period orbits than outer Oort cloud comets -- typical periods in the inner cloud are 2 to $5 \times 10^{5}$ years versus $4 \times 10^{6}$ years in the outer cloud -- and returning comets tend to be perturbed to even shorter period orbits, $\sim 10^{3}$ to $10^{5}$ years. They thus make many 
returns in a relatively short period of time. The temporal profile and fraction of surviving comets for a major cometary shower as found by Hut and Weissman (1985) is shown in Figure 7.

Fortunately, major cometary showers as a result of deep, $\sim 3 \times 10^{3} \mathrm{AU}$, penetrating stellar encounters are rare, occurring perhaps once every 3 to $5 \times 10^{8}$ years. Cometary showers can also result with a similar frequency, due to random encounters with GMCs, but with perhaps an order of magnitude less total flux into the planetary region (Morris and Muller, 1987). Lesser showers from more distant, but still penetrating stellar passages at heliocentric distances $\sim 10^{4} \mathrm{AU}$ occur somewhat more frequently, on the order of every $5 \times 10^{7}$ years. As a result, random cometary showers dominate the cometary contribution to cratering on the terrestrial planets (Weissman, 1990). Such showers have also been suggested as a possible cause of biological extinction events on the Earth (Hills, 1981). Cometary impacts are expected to account for approximately $30 \%$ of the craters larger than $10 \mathrm{~km}$ diameter on the Earth and Moon (Weissman, 1990).

Raup and Sepkoski (1984) suggested that biological extinction events on the Earth repeat with an approximately $26 \times 10^{6}$ year period, based on their statistical study of the fossil record. This led to several hypotheses that each invoked cometary showers as the cause of the extinctions. These hypotheses involved: 1) a dwarf companion star to the

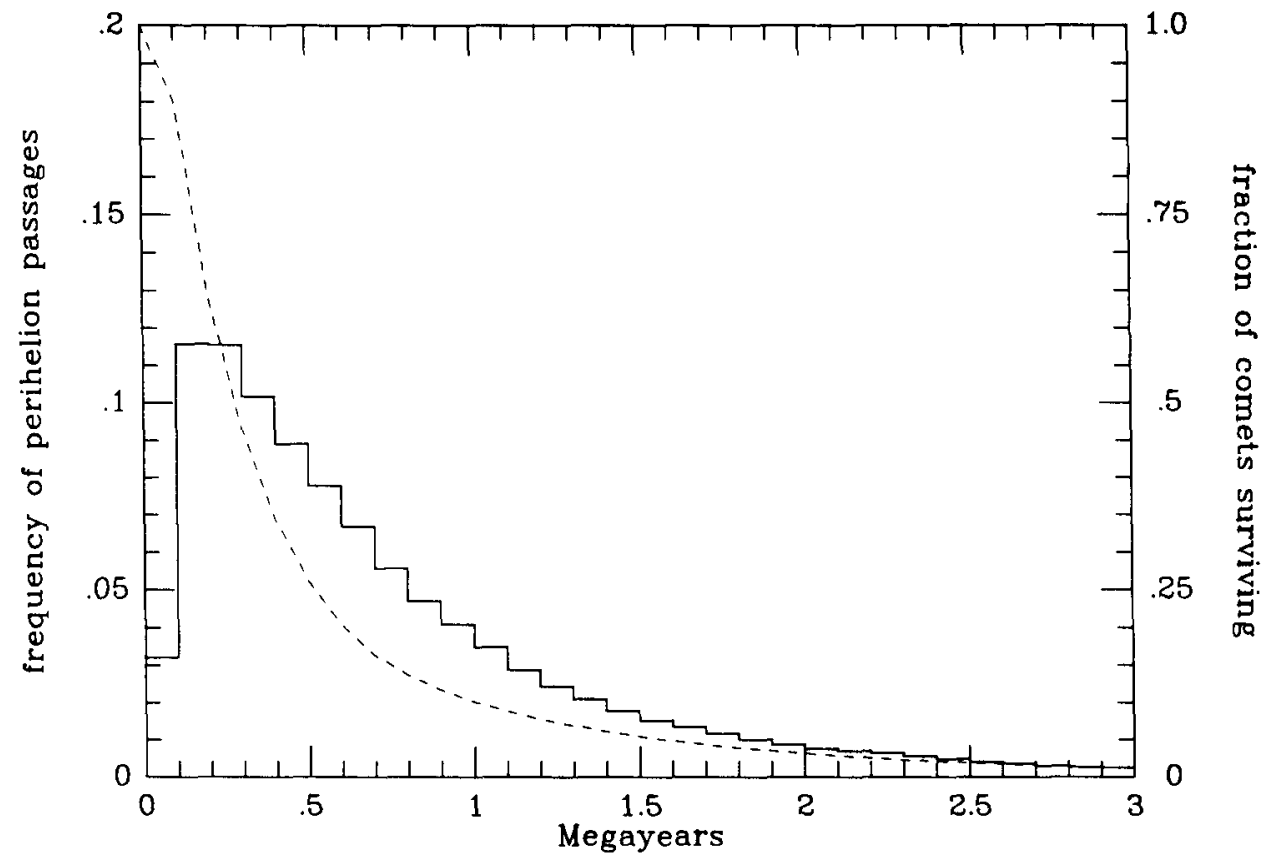

Figure 7. Dynamical evolution of a shower of comets from the inner Oort cloud due to a close, penetrating stellar passage, $3 \times 10^{3} \mathrm{AU}$ from the Sun. The solid histogram is the relative number of comets crossing the Earth's orbit versus time; the dashed curve is the fraction of the original shower comets still evolving in the system. On the order of $5 \times 10^{8}$ comets (brighter than $\mathrm{H}_{10}=11.0$ ) are expected to be thrown into Earth-crossing orbits by the star's perturbations. From Hut and Weissman (1985). 
Sun in a distant, eccentric, $26 \times 10^{6}$ year period orbit with its perihelion deep in the Oort cloud (Whitmire and Jackson, 1984; Davis et al., 1984); 2) a 10th planet circulating in a highly inclined, precessing orbit at about $150 \mathrm{AU}$ from the Sun, at the inner edge of the inner Oort cloud (Whitmire and Matese, 1985); or 3) the solar system's epicyclic motion above and below the galactic plane with a half-period of 32 to $33 \times 10^{6}$ years, with GMC encounters at galactic plane crossings (Rampino and Stothers, 1984). The apparent coincidence between galactic plane crossings by the solar system and terrestrial extinction boundaries had been pointed out originally by Innanen et al. (1978). The Sun's vertical motion was also suggested as the clock mechanism by Schwartz and James (1984), though they only speculated about the underlying physical mechanism leading to the extinctions.

A variety of dynamical problems have been identified with each of these hypotheses, and no evidence in support of any of them has been found. The orbit of the hypothetical Death Star is unstable, with an expected lifetime of $<10^{9}$ years (Hills, 1984; Torbett and Smoluchowski, 1984). The orbital period of the dwarf star should vary by about $10 \%$ per orbit and should random walk away from a fixed $26 \times 10^{6}$ year period (Weissman, 1986b). The precessing 10th planet, allegedly located in a cometary ring beyond the outer planets, produces comet showers broadly distributed in time and with too few comets to result in a high likelihood of terrestrial impacts (Weissman, 1986b; Tremaine, 1986). Because the scale height of GMCs above and below the galactic plane is almost the same as the maximum oscillation of the Sun and planetary system, the Oort cloud is almost as likely to encounter a GMC away from the plane as it would close to the galactic plane (Thaddeus and Chanan, 1985; Bahcall and Bahcall, 1985). As a result, no periodic signal would be derived from such encounters.

Questions have also been raised about the reality of the periodicity in the extinction record. Criticism has been made of the statistical techniques used to claim that the periodicity is significant (Hoffman, 1985), and of the accuracy of the dated tie-points in the geologic record, particularly prior to $140 \mathrm{Myr}$ ago (Shoemaker and Wolfe, 1986).

A criticism that bears on all periodic comet shower hypotheses is that the observed cratering record on the Earth and Moon does not show the expected number of craters that would result from intense periodic comet showers every 26 to $32 \times 10^{6}$ years (Weissman, 1990). This situation might be remedied if the population of the Oort cloud was a factor of four (or more) less than currently estimated, but that would also mean that showers would contain fewer comets, and that the expected number of impacts on the Earth would be reduced accordingly. In addition, there is little evidence of multiple impacts, i.e., multiple craters, iridium layers, tektite layers, etc., at extinction boundaries (Weissman, 1985b; Hut et al., 1987; Zhou and Kyte, 1988; Kyte, 1988, and references therein).

For example, the distribution of ages for dated craters on the Earth is shown in Figure 8 (from Weissman, 1990, based on data by Grieve, 1987). There is no significant clustering of ages that can be interpreted as evidence of a cometary shower, and no clustering of craters at the dates of the five major extinctions in the past $500 \mathrm{Myr}$. This is somewhat curious, since, even if periodic showers do not exist, at least one major random cometary shower would be expected in that time. Age dating of craters on the lunar maria, sometime in the future, may provide the data required to resolve these questions. 


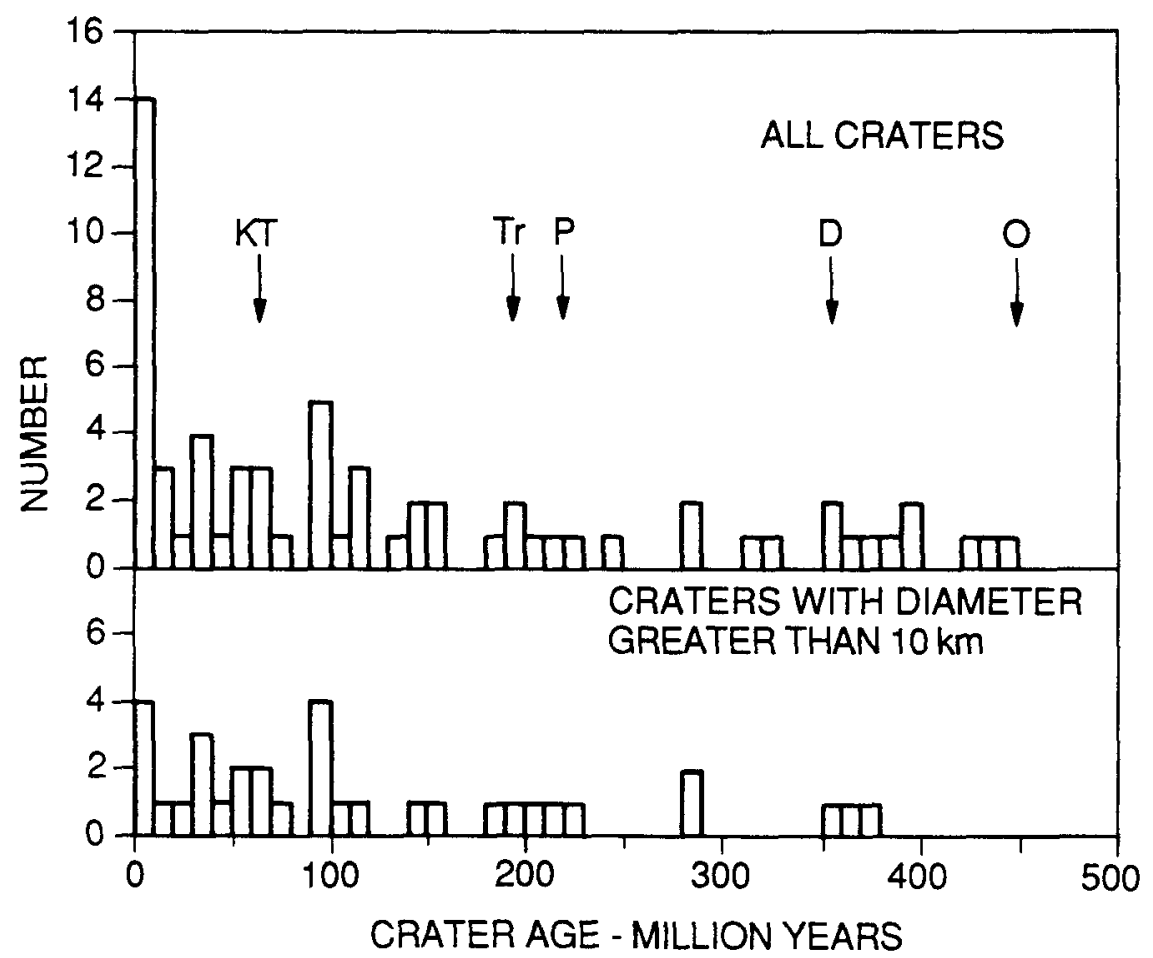

Figure 8. Histogram of ages of dated craters on the Earth based on data from Grieve (1987). Top, for all 65 dated craters, and bottom, for 33 dated craters with diameters $\geq 10 \mathrm{~km}$. There does not appear to be any significant correlation between the crater ages and the five major extinction events of the past $500 \mathrm{Myr}$, shown by the vertical arrows. In addition, there is little evidence for cometary showers, i.e., clusters of crater ages. The large number of craters in the most recent $10 \mathrm{Myr}$ interval is a selection effect; the youngest craters have not yet had time to degrade physically.

\section{The Kuiper Belt}

An interesting recent development has been the addition of a third component to the Oort cloud. New Monte Carlo simulations by Duncan et al. (1988) have shown that most of the short-period (SP) comets likely do not result from the dynamical evolution of LP comets. The LP comets tend to preserve their random inclinations as they evolve inward to short-period orbits as a result of planetary perturbations. But the observed distribution of inclinations for the SP comets is largely confined to direct orbits with inclinations $<30^{\circ}$. Comet Halley is a notable exception to this distribution; only five retrograde SP comets are known to exist.

Duncan et al. (1988) showed that a more plausible source for the SP comets is a flattened belt or ring of comets beyond Neptune. This belt, presumably a remnant of the original accretion disc of planetesimals in the solar nebula, was first suggested by Kuiper 
(1951), and was discussed by Cameron (1962, 1978) and Whipple (1964). It had been thought that such a belt might merge continuously into the inner Oort cloud. However, Duncan et al.'s work suggests that the two comet populations are dynamically distinct. The comets in the belt may have semimajor axes of $\sim 50$ to 500 AU (though the upper limit is entirely speculative), whereas inner Oort cloud comets likely have minimum semimajor axes of $\sim 2,000$ to 3,000 AU.

Fernandez (1980) and Bailey (1983) estimated that a comet belt beyond Neptune would be 300 times more efficient dynamically for producing SP comets than direct evolution of LP comets from the outer Oort cloud. Larger bodies on the order of the size of Ceres or perhaps Pluto are required to be circulating in the belt to perturb some of the comets into Neptune-crossing orbits occasionally, beginning their dynamical diffusion inward to short-period orbits.

The number of comets in the "Kuiper Belt," as Duncan et al. call it, is estimated to be on the order of $10^{8}$ to $10^{10}$ objects. Yeomans (1986) estimated an upper limit on the mass of any trans-Neptunian comet belt of $\sim 1.0 \mathrm{M}_{\oplus}$, based on the failure to detect any perturbations on the orbit of Comet Halley. A somewhat weaker upper limit of $5 \mathrm{M}_{\oplus}$ was set by Anderson and Standish (1986) based on tracking of the Pioneer 10 spacecraft; this method has the potential for setting stricter limits in the future as Pioneer 10 continues to move outward through the Kuiper Belt zone. Duncan et al. estimated a minimum total mass of $0.02 \mathrm{M}_{\oplus}$ was needed in the comet belt to maintain the supply of observed SP

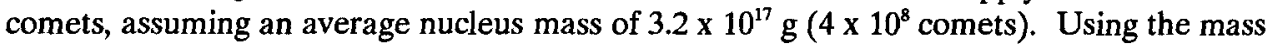
distribution shown in Figure 5, the same number of comets would have a total mass of $0.0026 \mathrm{M}_{\oplus}$.

\section{Physical Processing of Comets in the Oort Cloud}

It had generally been thought that the Oort cloud is a fairly benign storage location for the LP comets. The typical spacing between comets in the outer cloud is $\sim 15 \mathrm{AU}$, and in the inner cloud it is $\sim 1 \mathrm{AU}$. The typical temperature is that of interstellar space, $\leq$ $10 \mathrm{~K}$. However, it is now recognized that a variety of processes combine to act on the cometary nuclei, in particular, their surface layers, over the history of the solar system (Weissman, 1986c). These include: irradiation, sputtering, and polymerization by galactic cosmic rays (Johnson et al., 1987); heating by passing stars and nearby supernovae (Stern and Shull, 1988); gardening by debris impacts (Stern, 1988); and the accretion of interstellar dust and gas and accompanying erosion by hypervelocity dust impacts (Stern, 1986, 1990). In addition, the interiors of the nuclei may have been warmed by long-lived radionuclides (Lewis, 1971), and even melted if short-lived radionuclides such as ${ }^{26} \mathrm{Al}$ are there in similar abundances to those found for some primitive carbonaceous chondrites (Wallis, 1980; Prialnik and Bar-Nun, 1988).

The combined effects of these competing processes are not well understood. They may lead to the development of a permanent nonvolatile crust over the nucleus surface before it ever enters the planetary system, or they may simply result in a heterogeneous collection of unbound, highly processed materials in a well-gardened nucleus regolith. Surface heating may cause both loss and inward diffusion of the more volatile cometary species, while internal heating will also mobilize volatile species and could conceivably result in the nucleus core transitioning from amorphous to crystalline ice. 


\section{Extra-solar Oort Clouds and Interstellar Comets}

Presumably, the same processes that led to the formation of planetesimals and their ejection to form an Oort cloud around our planetary system can also occur around other forming stars. Because the expected dimensions of Oort clouds are so large, it may be possible to actually detect and resolve such comet clouds. One method would be to look for thermal radiation from dust created by collisions and sputtering in the cloud. Such an analysis of sky images at the four bandpasses of the Infrared Astronomical Satellite (IRAS) has been performed for 17 nearby stars by Stern et al. (1990a), looking for infrared excess in summed circular annuli around the primaries. However, no detections have been reported. This is as expected, since dust at Oort cloud distances will be very cold, and radiation pressure and collisions with interstellar dust and gas should rapidly sweep fine dust from the comet clouds (Stern, 1990).

Stern et al. (1990b) have also proposed searching for Oort clouds around red giant stars. These stars are sufficiently luminous that the comets in their Kuiper Belts, if they had them, would be actively sublimating at rates comparable to the gas production rates of comets at $1 \mathrm{AU}$ in our own solar system. Stern et al. have suggested that observed $\mathrm{OH}$ IR stars may be an example of this phenomena.

It is interesting to speculate on the fate of the many comets ejected to interstellar space in forming the Oort cloud and over its history. Dynamical ejection is the most common loss mechanism for comets in the cloud, either due to close stellar and GMC perturbations, or as a result of Jupiter perturbations during a pass through the planetary system.

No comet on a clearly interstellar trajectory has been observed passing through the planetary system. Sekanina (1976) showed that this fact sets an upper limit on the space density of interstellar comets of $6 \times 10^{-4} \mathrm{M}_{\odot} \mathrm{pc}^{-3} \approx 4 \times 10^{12}$ comets pc ${ }^{-3}$, using Sekanina's mean nucleus mass of $3 \times 10^{17} \mathrm{~g}$. For comparison, this is $\sim 300$ times less than the density of material in the solar neighborhood, $\sim 0.185 \mathrm{M}_{\odot} \mathrm{pc}^{\cdot 3}$ (Bahcall, 1984), so interstellar comets cannot contribute significantly to the "missing mass" problem in the galaxy. It is about half the density for comets in the outer Oort cloud, assuming a population of $10^{12}$ comets in a sphere of radius $10^{5} \mathrm{AU}$ centered on the Sun. Thus, the limit is not very strict.

It is also possible to compare the limit above with the estimated space density of interstellar comets, if it is assumed that all stars produce cometary clouds. Dynamical models estimate that between 3 (Fernandez and Ip, 1981) and 50 (Safronov, 1972) times as many comets are ejected by the proto-planets as are placed in the Oort cloud (though it can be shown that Safronov's estimate is extreme, as it assumes a very narrow range of semimajor axes for the Oort cloud). Another factor of two to three comes from the comets lost from the Oort cloud over the history of the solar system. Thus, taking a nominal current Oort cloud population of $7.0 \times 10^{12}$ comets, the solar system has ejected $\sim 6 \times 10^{13}$ to $10^{15}$ comets to interstellar space. Taking a mean volume per star in the solar neighborhood of $\sim 12 \mathrm{pc}^{3}$ (Allen, 1973), and assuming that all stars produce comet clouds, the predicted space density is $5 \times 10^{12}$ to $9 \times 10^{13}$ comets $\mathrm{pc}^{-3}$. This is 1.3 to 23 times the upper limit determined by Sekanina.

Since half of all stars form in multiple systems, and that process may prevent the formation of a protoplanetary disk leading to cometesimals (though that conjecture has not actually been demonstrated), the factor of 1.3 excess is likely not a problem. However, a factor of 23 excess is not consistent with the Oort cloud models and population estimates presented here, and thus represents a problem that clearly needs to be resolved. As noted 
above, at least a factor of two (and probably four) reduction can be obtained by simply assuming a wider range of Oort cloud semimajor axes than in Safronov's work. The remaining discrepancy clearly merits further study.

The same problem was recently studied by McGlynn and Chapman (1989), who suggested that at least six interstellar comets should have been observed passing within $2 \mathrm{AU}$ of the Sun in the past 150 years, even after accounting for the fact that only $7 \%$ of all long-period comets passing within $2 \mathrm{AU}$ are expected to be discovered. Their estimate was based on an average ejected population of $10^{14}$ comets per star. McGlynn and Chapman's estimate of six observable interstellar comets appears to be high by about a factor of two as compared with the Sekanina upper limit given above. The reason for this discrepancy is not clear, though the difference may not be significant.

\section{The Sun's Galactic Motion}

Most models of the Oort cloud are evaluated assuming the Sun's current position in the Galaxy, its current velocity relative to the Local Standard of Rest (LSR), and its current perturbing environment of stars, GMCs, and the galactic gravitational field. However, the Sun's motion has likely varied considerably over the history of the solar system, precisely because of random encounters with GMCs. Wielen (1977) showed that the observed velocity dispersions for stars of different stellar classes (and hence, different ages) can be explained by a model that assumed formation in dense interstellar clouds with initially small random velocities on the order of a few kilometers per second. and subsequent acceleration by random GMC encounters throughout their histories.

An interesting aspect of this problem is to compare the Sun's motion with that typical for other G-type stars. The Sun's velocity ellipsoid has dimensions of $9 \times 12 \times 7 \mathrm{~km} \mathrm{~s}^{-1}$ relative to the LSR, where the velocity components are radial with respect to the galactic nucleus, perpendicular to the radial vector in the galactic plane, and perpendicular to the galactic plane (Mihalas and Binney, 1981). The Sun's net velocity relative to the LSR is $16.5 \mathrm{~km} \mathrm{~s}^{-1}$. In general terms, the Sun is moving "inward" towards galactic periapsis, and "upwards," having recently passed through the galactic plane. In comparison, the mean velocity ellipsoid for G0 stars in the Sun's vicinity (the Sun is type G2) is $26 \times 18 \times 20 \mathrm{~km}$ $\mathrm{s}^{-1}$, for a total velocity of $37.4 \mathrm{~km} \mathrm{~s}^{-1}$. For G5 stars, the ellipsoid is similar: $32 \times 17 \times 15$ $\mathrm{km} \mathrm{s}^{-1}$, for a total velocity of $39.2 \mathrm{~km} \mathrm{~s}^{-1}$.

Thus, the Sun's motion is anomalously slow as compared with that of typical stars of its type and age; this was pointed out by Hut and Tremaine (1985) in their evaluation of perturbations by GMCs on the Oort cloud. It is likely that the Sun has random walked in velocity over its history, and has, for some substantial fraction of its past, moved faster relative to the LSR. However, the fact that the Sun is currently moving relatively slowly constrains its past history, such that it has likely not moved exceedingly fast in the past. Hut and Tremaine estimated that the root-mean-square (rms) velocity for the Sun over its history, based on an analogy with Brownian motion, is $18 \mathrm{~km} \mathrm{~s}^{-1}$, less than $2 \mathrm{~km} \mathrm{~s}^{-1}$ greater than its current velocity relative to the LSR.

Although the Sun's current motion is close to its long-term average, the rms velocity for the Sun's current galactic orbit is only $11.7 \mathrm{~km} \mathrm{~s}^{-1}$. The Sun is moving faster than its average right now because it is near the periapse of its galactic orbit (Innanen et al., 1978) and because it has just passed through the galactic plane. Thus, on the average, the Sun 
has moved faster and farther out of the galactic plane in the past.

The consequences of this are interesting. As pointed out by Hut and Tremaine (1985), there are two important effects. First, because the Sun moves faster, the effect of individual perturbations by stars and/or GMCs is decreased. The magnitude of the impulse perturbation goes as $1 / \mathrm{V}$. The frequency of encounters will increase as $\mathrm{V}$, but since individual impulses will add randomly, the impulses will sum as $\mathrm{V}^{-1 / 2}$. As a result, a higher encounter velocity means lower net perturbations on the Oort cloud.

The second important effect is the motion of the Sun out of perturbing regions, both in terms of its vertical distance out of the galactic plane, and its radial excursions outward in the galaxy. This effect was studied by Hut and Tremaine, who concluded that total perturbations were diminished by factors of 0.5 and 0.7 for $\mathrm{z}$-motion and radial motion, respectively. Bailey (1983) also considered this problem and found correction factors of 0.4 and 1.0 for z-motion and radial motion, respectively. Thus, estimates for the total perturbations on the Oort cloud based on the Sun's current galactic position tend to overestimate the perturbations by a factor of 2.5 to 2.8 . This is one area that certainly deserves additional study in the future.

\section{Discussion}

Our view of the Oort cloud has evolved considerably since it was first proposed in 1950. Much of that evolution has come in the past decade, as a result of the availability of sufficient computing power to simulate the chaotic dynamical evolution of large numbers of hypothetical comets under a combination of perturbers. Another important factor has been a number of speculative hypotheses, often involving catastrophic events, which have not proven to be correct, but which nonetheless have motivated researchers to improve their modeling and understanding of the dynamics of comets in the cloud. In addition, if cometary clouds are a natural result of star and planet formation, we are approaching a time and a level of understanding when such clouds may be detectable. The Hubble Space Telescope, the Infrared Space Observatory, and other planned orbiting telescopes may soon provide the first conclusive evidence of Oort clouds around other stars, certainly one of the most exciting discoveries that we can anticipate. The existence of Oort clouds would strongly imply the existence of large planets, necessary to eject the proto-comets to Oort cloud distances.

But our understanding of the Oort cloud is still far from complete. The observed distributions of orbital elements in galactic coordinates do not precisely match the expected distributions from Monte Carlo simulation modeling of the Oort cloud. The average aphelion distance of dynamically new comets from the cloud is somewhat less than is expected from studies of the combined effects of Oort cloud perturbers. As pointed out in the previous section, one must also consider the consequences of including a variable solar motion in the models, a motion that likely has the solar system moving faster relative to its perturbers in the past, and moving farther out of the galactic plane than its present epicyclic motion allows.

The Oort cloud has "grown" in number of comets, total mass, and complexity over the past 10 years. But the central concept of a roughly spherical comet cloud surrounding the planetary system and stretching halfway to the nearest stars has remained remarkably 
intact. It will be interesting to see what changes to the current view are brought about by another decade of study.

Acknowledgment. It is a pleasure to thank Julia Heisler for several long and fruitful discussions on Oort cloud dynamics. The author also wishes to thank Mark Bailey, Philip Campbell, Jan Oort, Alan Stern, and Don Yeomans for their reviews of an earlier draft of this paper. This work was supported by the NASA Planetary Geology and Geophysics Program, and was performed at the Jet Propulsion Laboratory under a contract with the National Aeronautics and Space Administration.

\section{References}

Allen, C. W. (1973) Astrophysical Quantities, Athlone Press, London, $310 \mathrm{pp.}$

Anderson, J. D., and Standish, E. M. Jr. (1986) Dynamical evidence for Planet X. In The Galaxy and the Solar System, eds. R. Smoluchowski, J. N. Bahcall, and M. S. Matthews, Univ. Arizona Press, Tucson, pp. 286-296.

Antonov, V. A., and Latyshev, I. N. (1972) Determination of the form of the Oort cometary cloud as the Hills surface in the Galactic field. In The Motion, Evolution of Orbits, and Origin of Comets, eds. G. A. Chebotarev, E. I. Kazimirchak-Polonskaya, and B. G. Marsden, D. Reidel, Dordrecht, pp. 341-345.

Bahcall, J. N. (1984) Self-consistent determination of the total amount of matter near the Sun. Astrophys. J. 276, 169-181.

Bahcall, J. N., and Bahcall, S. (1985) The Sun's motion perpendicular to the galactic plane. Nature 316, 706-708.

Bailey, M. E. (1983) Comets, planet X, and the orbit of Neptune. Nature 302, 399-400. Bailey, M. E. (1984) The steady-state 1/a distribution and the problem of cometary fading. Mon. Not. Roy. Astron. Soc. 211, 347-368.

Bailey, M. E. (1986) The mean energy transfer rate to comets in the Oort cloud and implications for cometary origins. Mon. Not. Roy. Astron. Soc. 218, 1-30.

Bailey, M. E., and Stagg, C. R. (1988) Cratering constraints on the inner Oort cloud: Steady-state models. Mon. Not. Roy. Astron. Soc. 235, 1-35.

Biermann, L. (1978) Dense interstellar clouds and comets. In Astronomical Papers Dedicated to Bengt Stromgren, eds. A. Reiz and T. Anderson, Copenhagen Obs., pp. 327335.

Brin, G. D., and Mendis, D. A. (1979) Dust release and mantle development in comets. Astrophys. J. 229, 402-408.

Byl, J. (1983) Galactic perturbations on nearly parabolic cometary orbits. Moon \& Planets 29, 121-137.

Cameron, A. G. W. (1962) The formation of the sun and planets. Icarus 1, 13-69.

Cameron, A. G. W. (1978) The primitive solar accretion disc and the formation of the planets. In The Origin of the Solar System, ed. S. F. Dermott, John Wiley \& Sons, New York, pp. 49-75.

Clube, S. V. M., and Napier, W. M. (1982) Spiral arms, comets and terrestrial catastrophism. Quart. J. Roy. Astron. Soc. 23, 45-66.

Clube, S. V. M., and Napier, W. M. (1984) Comet capture from molecular clouds: A 
dynamical constraint on star and planet formation. Mon. Not. Roy. Astron. Soc. 208, 575-588.

Davis, M., Hut, P., and Muller, R. A. (1984) Extinction of species by periodic comet showers. Nature 308, 715-717.

Delsemme, A. H. (1987) Galactic tides affect the Oort cloud: An observational confirmation. Astron. \& Astrophys. 187, 913-918.

Duncan, M., Quinn, T., and Tremaine, S. (1987) The formation and extent of the solar system comet cloud. Astron. J. 94, 1330-1338.

Duncan, M., Quinn, T., and Tremaine, S. (1988) The origin of short-period comets. Astrophys. J. 328, L69-L73.

Everhart, E. (1967) Intrinsic distributions of cometary perihelia and magnitudes. Astron. J. 72, 1002-1011.

Fernandez, J. A. (1980) On the existence of a comet belt beyond Neptune. Mon. Not. Roy. Astron. Soc. 192, 481-491.

Fernandez, J. A. (1982) Dynamical aspects of the origin of comets. Astron J. 87, 13181332.

Fernandez, J. A. (1990) Statistical and evolutionary aspects of cometary orbits. In Comets in the Post-Halley Era, eds. R. L. Newburn Jr., J. Rahe, and M. M. Neugebauer, Kluwer, Dordrecht, in press.

Fernandez, J. A., and Ip, W.-H. (1981) Dynamical evolution of a cometary swarm in the outer planetary region. Icarus 47, 470-479.

Fernandez, J. A., and Ip, W.-H. (1987) Time dependent injection of Oort cloud comets into Earth-crossing orbits. Icarus 71, 46-56.

Grieve, R. A. F. (1987) Terrestrial impact structures. Ann. Rev. Earth \& Planet Sci. 17, 245-270.

Halley, E. (1705) A Synopsis of the Astronomy of Comets. London, $24 \mathrm{pp}$.

Harrington, R. S. (1985) Implications of the observed distributions of very long-period comet orbits. Icarus 61, 60-62.

Heisler, J., and Tremaine, S. (1986) The influence of the galactic tidal field on the Oort comet cloud. Icarus 65, 13-26.

Heisler, J., Tremaine, S., and Alcock, C. (1987) The frequency and intensity of comet showers from the Oort cloud. Icarus 70, 269-288.

Heisler, J. (1990) Monte Carlo simulations of the Oort comet cloud. Icarus, submitted.

Hills, J. G. (1981) Comet showers and the steady-state infall of comets from the Oort cloud. Astron. J. 86, 1730-1740.

Hills, J. G. (1984) Dynamical constraints on the mass and perihelion distance of Nemesis and the stability of its orbit. Nature 311, 636-638.

Hoffman, A. (1985) Patterns of family extinction depend on definition and geologic timescale. Nature 315, 659-662.

Hut, P., and Weissman, P. R. (1985) Dynamical evolution of cometary showers. Bull. Amer. Astron. Soc. 17, 690 (abstract).

Hut, P., and Tremaine, S. (1985) Have interstellar clouds disrupted the Oort comet cloud? Astron. J. 90, 1548-1557.

Hut, P., Alvarez, W., Elder, W. P., Hanson, T., Kauffmann, E. G., Keller, G., Shoemaker, E. M., and Weissman, P. R. (1987) Comet showers as a cause of stepwise extinctions. Nature 329, 118-126.

Innanen, K. A., Patrick, A. T., and Duley, W. W. (1978) The interaction of the spiral 
density wave and the Sun's galactic orbit. Astrophys. Space Sci. 57, 511-515.

Kyte, F. T. (1988) The extraterrestrial component in marine sediments: Description and interpretation. Paleoceanography 3, 235-247.

Johnson, R. E., Cooper, J. F., Lanzerotti, L. J., and Strazzula, G. (1987) Radiation formation of a non-volatile comet crust. Astron. Astrophys. 187, 889-892.

Kuiper, G. P., 1951. On the origin of the solar system. In Astrophysics, ed. J. A. Hynek, McGraw Hill, New York, pp. 357-424.

Marochnik, L. S., Mukhin, L. M., and Sagdeev, R. Z. (1988) Estimates of mass and angular momentum in the Oort cloud. Science 242, 547-550.

Marsden, B. G., Sekanina, Z., and Yeomans, D. K. (1973) Comets and nongravitational forces. V. Astron. J. 78, 211-225.

Marsden, B. G., Sekanina, Z., and Everhart, E. (1978) New osculating orbits for 110 comets and the analysis of the original orbits of 200 comets. Astron J. 83, 64-71.

McGlynn, T. A. and Chapman, R. D. (1989) On the nondetection of extrasolar comets. Astrophys. J. 346, L105-108.

Mihalas, D., and Binney, J. (1981) Galactic Astronomy, Structure and Kinematics, W. H. Freeman, San Francisco, 597 pp.

Morris, D. E., and Muller, R. A. (1986) Tidal gravitational forces: The infall of "new" comets and comet showers. Icarus 65, 1-12.

Nezhinskij, E. M. (1972) On the stability of the Oort cloud. In The Motion, Evolution of Orbits, and Origin of Comets, eds. G. A. Chebotarev, E. I. Kazimirchak-Polonskaya, and B. G. Marsden, D. Reidel, Dordrecht, pp. 335-340.

Oort, J. H. (1950) The structure of the cloud of comets surrounding the solar system and a hypothesis concerning its origin. Bull. Astron. Inst. Neth. 11, 91-110.

Oort, J. H., and Schmidt, M. (1951) Differences between new and old comets. Bull. Astron. Inst. Neth. 11, 259-269.

Öpik, E. (1932) Note on stellar perturbations of nearly parabolic orbits. Proc. Amer. Acad. Arts. \& Sci. 67, 169-183.

Peale, S. J. (1989) On the density of Halley's comet. Icarus 82, 36-49.

Prialnik, D., and Bar-Nun, A. (1987) On the evolution and activity of cometary nuclei. Astrophys. J. 313, 893-905.

Rampino, M. R., and Stothers, R. B. (1984) Terrestrial mass extinctions, cometary impacts, and the sun's motion perpendicular to the galactic plane. Nature 308, 709-712.

Raup, D. M., and Sepkoski, J. J. (1984) Periodicity of extinctions in the geologic past. Proc. Natl. Acad. Sci. USA 81, 801-805.

Rickman, H. (1986) Masses and densities of comets Halley and Kopff. In Comet Nucleus Sample Return, ESA SP-249, pp. 195-205.

Safronov, V. S. (1972) Evolution of the Protoplanetary Cloud and Formation of the Earth and Planets, NASA TT-F-677 (Nauka Press, Moscow, 1969).

Sagdeev, R. Z., Elyasberg, P. E., and Moroz, V. I. (1987) Is the nucleus of comet Halley a low density body? Nature 331, 240-242.

Schwartz, R. D., and James, P. B. (1984) Periodic mass extinctions and the Sun's oscillation about the galactic plane. Nature 308, 712-713.

Sekanina, Z. (1976) A probability of encounter with interstellar comets and the likelihood of their existence. Icarus 27, 123-133.

Shoemaker, E. M., and Wolfe, R. F. (1984) Evolution of the Uranus-Neptune planetesimal swarm. Lunar Planet Sci. Conf. XV, 780-781 (abstract). 
Shoemaker, E. M., and Wolfe, R. F. (1986) Mass extinctions, crater ages, and comet showers. In The Galaxy and the Solar System, eds. R. Smoluchowski, J. N. Bahcall, and M. S. Matthews, Univ. Arizona Press, Tucson, pp. 338-386.

Smoluchowski, R., and Torbett, M. (1984) The boundary of the solar system. Nature 311, 38-39.

Stern, S. A. (1986) The effects of mechanical interaction between the interstellar medium and comets. Icarus $68,276-283$.

Stern, S. A. (1988) Collisions in the Oort cloud. Icarus 73, 499-507.

Stern, S. A. (1990) ISM induced erosion and gas dynamical drag in the Oort cloud. Icarus, in press.

Stern, S. A., and Shull, J. M. (1988) The thermal evolution of comets in the Oort cloud by stars and supernovae. Nature 332, 407-411.

Stern, S. A., Stocke, J., and Weissman, P. R. (1990) An IRAS search for extra-solar Oort clouds. Icarus, submitted.

Stern, S. A., Shull, M. J., and Brandt, J. C. (1990b) The evolution and detectability of comet clouds during post main sequence stellar evolution. Nature, in press.

Thaddeus, P., and Chanan, G. A. (1985) Cometary impacts, molecular clouds, and the motion of the Sun perpendicular to the galactic plane. Nature 314, 73-75.

Torbett, M. V., and Smoluchowski, R. (1984) Orbital stability of an unseen solar companion linked to periodic extinction events. Nature 311, 641-642.

Tremaine, S. (1986) Is there evidence for a solar companion. In The Galaxy and the Solar System, eds. R. Smoluchowski, J. N. Bahcall, and M. S. Matthews, Univ. Arizona Press, Tucson, pp. 409-416.

Valtonen, M. J. (1983) On the capture of comets into the inner solar system. Observatory 103, 1-4.

Valtonen, M. J. and Innanen, K. A. (1982) The capture of interstellar comets. Astrophys. J. 255, 307-315.

van Woerkom, A. F. F. (1948) On the origin of comets. Bull. Astron. Inst. Neth. 10, 445472.

Wallis, M. K. (1980) Radiogenic melting of primordial comet interiors. Nature 284, 431432.

Weissman, P. R. (1979) Physical and dynamical evolution of long-period comets. In Dynamics of the Solar System, ed. R. L. Duncombe, D. Reidel, Dordrecht, pp. 277-282.

Weissman, P. R. (1980) Stellar perturbations of the cometary cloud. Nature 288, 242-243.

Weissman, P. R. (1982) Dynamical history of the Oort cloud. In Comets, ed. L. L. Wilkening, Univ. Arizona Press, Tucson, pp. 637-658.

Weissman, P. R. (1985a) Dynamical evolution of the Oort cloud. In Dynamics of Comets: Their Origin and Evolution, eds. A. Carusi and G. B. Valsecchi, D. Reidel, Dordrecht, pp. 87-96.

Weissman, P. R. (1985b) Terrestrial impactors at geologic boundary events: Comets or asteroids? Nature 314, 517-518.

Weissman, P. R. (1986a) The mass of the Oort cloud: A post Halley reassessment. Bull. Amer. Astron. Soc. 18, 799 (abstract).

Weissman, P. R. (1986b) The Oort cloud and the galaxy: Dynamical interactions. In The Galaxy and the Solar System, eds. R. Smoluchowski, J. N. Bahcall, and M. S. Matthews, Univ. Arizona Press, Tucson, pp. 204-237.

Weissman, P. R. (1986c) Are cometary nuclei really pristine? In The Comet Nucleus 
Sample Return Mission, ESA SP-249, pp. 15-25.

Weissman, P. R. (1990) The cometary impactor flux at the Earth. In Global Catastrophes in Earth History, GSA Special Paper 247, eds. V. Sharpton and P. Ward, in press.

Whipple, F. L. (1964) The history of the solar system. Proc. Natl. Acad. Sci. USA 51, 711718.

Whitmire, D. P., and Jackson, A. A. (1984) Are periodic mass extinctions driven by a distant solar companion? Nature 308, 713-715.

Whitmire, D. P., and Matese, J. J. (1985) Periodic comet showers and planet X. Nature 313, 36-38.

Wielen, R. (1977) The diffusion of stellar orbits derived from the observed age dependence of the velocity dispersions. Astron. Astrophys. 60, 263-275.

Yeomans, D. K. (1986) Physical interpretations from the motions of comets Halley and Giacobini-Zinner. In 20th ESLAB Symposium on the Exploration of Halley's Comet, eds. B. Battrick, E. J. Rolfe, and R. Reinhard, ESA SP-250, 2, 419-425.

Zhou, L., and Kyte, F. T. (1988) The Permian-Triassic boundary event: A geochemical study of three Chinese sections. Earth \& Planet. Sci. Lett. 90, 411-421. 